\title{
Detecting Silent Data Corruption for Extreme-Scale MPI Applications
}

\author{
Leonardo Bautista-Gomez \\ Argonne National Laboratory \\ leobago@anl.gov
}

\author{
Franck Cappello \\ Argonne National Laboratory \\ cappello@mcs.anl.gov
}

\begin{abstract}
Next-generation supercomputers are expected to have more components and, at the same time, consume several times less energy per operation. These trends are pushing supercomputer construction to the limits of miniaturization and energy-saving strategies. Consequently, the number of soft errors is expected to increase dramatically in the coming years. While mechanisms are in place to correct or at least detect some soft errors, a significant percentage of those errors pass unnoticed by the hardware. Such silent errors are extremely damaging because they can make applications silently produce wrong results. In this work we propose a technique that leverages certain properties of high-performance computing applications in order to detect silent errors at the application level. Our technique detects corruption based solely on the behavior of the application datasets and is applicationagnostic. We propose multiple corruption detectors, and we couple them to work together in a fashion transparent to the user. We demonstrate that this strategy can detect over $80 \%$ of corruptions, while incurring less than $1 \%$ of overhead. We show that the false positive rate is less than $1 \%$ and that when multi-bit corruptions are taken into account, the detection recall increases to over $95 \%$.
\end{abstract}

\section{Categories and Subject Descriptors}

Computer systems organization [Dependable fault-tolerant systems and networks]: Reliability

\section{Keywords}

High-performance computing, supercomputers, fault tolerance, silent data corruption, soft errors, anomaly detection

\section{INTRODUCTION}

Science applications require ever-larger machines to solve ever more complex problems. While future machines promise to tackle these problems, they are also raising new challenges. Both the transistor size and the energy consumption of

Publication rights licensed to ACM. ACM acknowledges that this contribution was authored or co-authored by an employee, contractor or affiliate of the United States government. As such, the Government retains a nonexclusive, royalty-free right to publish or reproduce this article, or to allow others to do so, for Government purposes only.

EuroMPI'15, September 21 - 23, 2015, Bordeaux, France

(C) 2015 Copyright held by the owner/author(s). Publication rights licensed to ACM. ACM ISBN 978-1-4503-3795-3/15/09 . .\$15.00

DOI: http://dx.doi.org/10.1145/2802658.2802665 future systems must be significantly reduced, steps that might dramatically impact the soft error (i.e., transient errors) rate (SER) according to recent studies [15].

Dynamic random memory access (DRAM) devices have been intensively protected against soft errors through errorcorrecting codes (ECCs) because they have the largest share of the susceptible surface on high-end computers. However, although ECC works well for DRAM, not all parts of the system are ECC protected: in particular, logic units and registers inside the processing units are usually not ECC protected because of the space, time, and energy cost that ECC requires to work at low level. Historically, the SER of central processing units was minimized through a technique called radiation hardening, which consists of increasing the capacitance of circuit nodes in order to increase the critical charge needed to change the logic level. Unfortunately, this technique involves increasing either the size or the energy consumption of the components, which is likely to be prohibitively expensive at extreme scale. Without such a technique, however, we can expect that a non-negligible percentage of soft errors could pass undetected by the hardware, corrupting the numerical data of scientific applications. This situation is called silent data corruption (SDC).

The datasets produced by high-performance computing (HPC) applications have characteristics that reflect the properties of the underlining physical phenomena that those applications seek to model. We propose to leverage the spatial and temporal behavior of these datasets in order to predict the normal evolution of the datasets. With this approach, SDCs will push the corrupted data point out of the expected range of normal values, making it an outlier.

The contributions of this work are summarized as follows.

- We study the propagation of corruption on HPC applications and identify negligible corruptions.

- We propose four detectors that detect anomalies based on the temporal and spatial smoothness of the datasets.

- We propose a dynamic elastic error learning technique that decreases the false positive rate to less than $1 \%$.

- We propose a fuzzy logic module that integrates the four detectors and returns an outlier score to the application so that different mitigation technique can be applied depending on the score.

- We implement this technique and optimize it to achieve less than $1 \%$ of overhead on the application. 


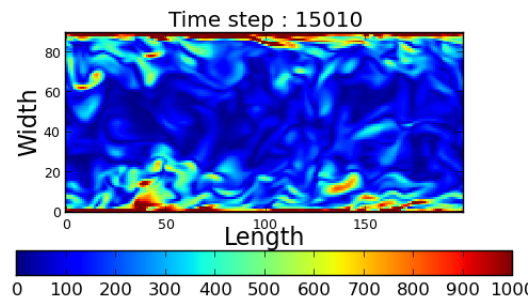

(a) Vorticity in turbulent fluid

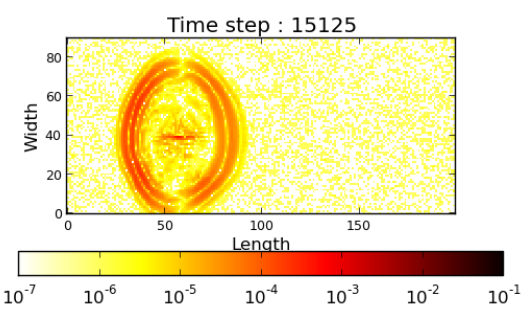

(b) Error propagation over the domain

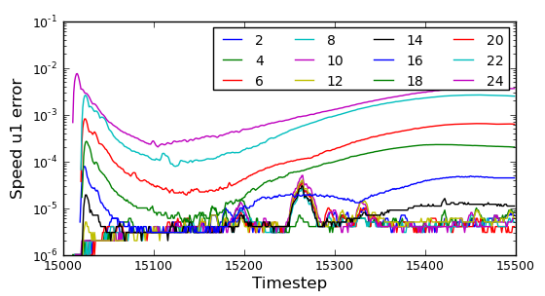

(c) Maximum deviation after corruption

Figure 1: Study of corruption propagation on a turbulence code for compressible gas.

- We demonstrate that, our detector achieves over $80 \%$ SDC coverage on a turbulent code and over 95\% SDC coverage when counting multi-bit errors.

The rest of this article is organized as follows. Section 2 presents our corruption propagation study. Section 3 introduces our proposed detectors and the fuzzy logic module. Section 4 shows our evaluation, including the corruption propagation analysis and the detectors' performance. Section 5 presents related work. Section 6 summarizes our work and presents some ideas for future research.

\section{CORRUPTION IN HPC APPLICATIONS}

We propose a novel method to detect SDC in scientific HPC applications. Data corruption can happen for different reasons and can be manifested in multiple ways leading to many possible outcomes. To narrow down the scope of this research, we introduce a fault model and describe the type of corruptions that our model is intended to handle.

\subsection{Fault Model}

The main challenge of dealing with SDC is the silent character of those errors. By silent one usually means that the fault escaped the hardware fault tolerance mechanisms and managed to propagate as a corruption without being detected by the hardware. Many of these faults, however, can corrupt control variables in the software, forcing the application to terminate (i.e., a loop counter out of range). These errors are silent to the hardware but not to the software stack. Thus, they can be treated as a fail-stop failures, and the application can be restarted from a previous checkpoint. In this work we focus only on errors corrupting non control variables, that is, large floating-point datasets that represent the majority of the memory allocated by the application and the majority of the data that moves between memory and CPU and that travels between MPI ranks.

We make no assumptions about the location of the corruptions. For instance, we assume that errors can happen inside the floating-point unit (FPU); thus one cannot use correcting codes to protect the data, because the output of an FPU operation is expected to be different from the input. In addition, we make no assumptions about the cause of the corruption, it could be due to cosmic rays, hardware damage, or even an intermittent bug. For this reason, we also do not make any assumption about the number of bits that get corrupted when these errors happen. In summary, we focus on soft errors that corrupt one or multiple bits of a floating-point dataset silently and that occur anywhere in the system regardless of their origin.

\subsection{Corruption Propagation Study}

An important point to analyze while studying SDC is the used-expected accuracy. Among the errors of the fault model just introduced, some SDCs will produce a divergence that is smaller than the error acceptable by the user. Hence, they can be safely neglected. Indeed, errors that are too small will not produce a significant impact on the final result of the application, and dedicating resources to detect such SDCs would be wasteful. An accurate estimation of the coverage of an SDC detector therefre is essential. We study the propagation of corruption inside an HPC application to expose this phenomenon, and to show which type of SDCs we target in this research.

We study one of the most challenging applications in this context: a turbulent flow in a 3D duct modeled as a large eddy simulation using a two-stage time-differencing scheme based on higher accuracy for compressible gas using NavierStokes equations. This model of turbulence is well known in the scientific community and is widely used in computational fluid dynamics (CFD). It represents a large set of HPC applications, ranging from weather prediction to aerospace engineering. Turbulence simulations are well known to have a chaotic and hard-to-predict behavior. For this simulation, the 3D duct is divided in $N$ sections along the length (x axis) of the duct, where $N$ corresponds to the number of MPI ranks in the simulation.

Figure 1(a) shows the vorticity of the fluid on a $2 \mathrm{D}$ cut of the 3D duct, aggregating data from all MPI processes in the simulation (total length of the duct). However, the vorticity is computed from the velocity fields in the three axes (for visualization) and is never stored in memory. An error deviation observed in the vorticity plot is the consequence of a corruption in one of the velocity fields. We injected a corruption in the velocity field $(u 1)$ following the $\mathrm{x}$ axis, in the grid point $40 X 40$. Figure 1 (b) plots the deviation a hundred time steps after corrupting the grid point $40 X 40$. We use a logarithmic color scale to show the magnitude of the deviation in the different regions of the domain. As we can observe, in only a hundred iterations the corruption has already propagated across the entire domain, and it shows a particularly high deviation in a region with a wave shape that has as its origin the corrupted grid point. Although the origin of the corruption was in the grid point $40 X 40$, the fluid has moved in those hundred time steps, and the corruption wave has as epicenter about 20 grid points to the right, which happens to be located in an MPI rank different from the one where the corruption was originally injected.

To study the propagation of corruption after an SDC, we performed the following experiment. First, we launched a 
turbulent flow simulation starting from the initial conditions and let it run for 15,000 time steps, while checkpointing every 500 time steps, with the last checkpoint taken at iteration 15,000. The purpose was to let the gas reach a relatively high level of turbulence. Then, we restarted the execution from the last checkpoint (i.e., time step 15,000), and we recorded the datasets of the execution at each time step for a corruption-free execution. We repeated the same corruption-free execution several times and confirmed that for each time step, all datasets are binary-identical between non corrupted executions. Then, we repeated the same experiment (restarting from time step 15,000) but this time injecting one bit-flip at bit position $p$ for $p$ in $\{2,4,6,8,10$, $12,14,16,18,20,22,24\}$, where 32 is the sign bit of the IEEE floating-point representation [14], the bits 24 to 31 are the exponent, and the remaining bits are the mantissa. For each experiment we injected the bit-flip in the first twenty time steps (i.e., before time step 15,020) and let it run for 500 iterations. After all the corruption experiments were done, we computed for each experiment and for each time step the difference between the corrupted dataset and the corruption-free dataset. This calculation generated about 4,000 images similar to Figure 1(b). To summarize those 4,000 images we plotted the maximum deviation at each time step for all the corruption experiments. The results are shown in Figure 1(c).

As we can see, during the first ten time steps or so, no corrupted data occurs. When the bit-flip is injected, we observe a sudden jump with a magnitude exponentially proportional to the bit-flip position (as can be read from the legend), which is consistent with the floating-point representation. In addition, we notice that immediately after the corruption jump, the deviation starts to decrease. This decrease is due to a smoothening effect that takes place when the non corrupted data interacts with the corrupted data. However, the same influence can go in the other direction. For instance, looking at the following hundred time steps, we notice that the high deviation wave bounces in the walls of the duct and continues propagating in other directions. The reflected wave then interacts with the other parts of the wave that is just arriving at the wall, generating an error amplification effect, which we see happening after time step 15,150 . The deviation then stabilizes around time step 15,400 and remains stable until the end of the execution.

As these results show, corruptions in bit position 12 and below generate a numerical deviation smaller than $10^{-4}$. Such insignificant corruptions can be neglected because they do not have a meaningful impact on the final results of the simulation. Nonetheless, corruptions in bit position 13 and above show important error deviations that cannot be neglected and that should be detected by the SDC detectors.

\section{UNSUPERVISED ANOMALY DETECTOR}

We propose to use an unsupervised anomaly detection approach to trigger alerts when SDC is suspected during the execution of extreme-scale scientific applications. We believe that a strategy based on data monitoring is an interesting path to explore for several reasons. First, such an approach is independent of the underlying algorithm and therefore more general than algorithm-based techniques. Second, one can develop lightweight data-monitoring techniques that impose a low overhead on the application (see Section 4.2) compared to that from expensive techniques such as double and triple redundancy. Third, unsupervised data monitoring can be offered by the runtime in a fashion transparent to the user. Finally, by operating at the application level (as opposed to hardware level), we have more information about which dataset need to be protected and their internal structure, hence improving detection efficiency.

\subsection{Cluster-Based Detection}

We monitor the application datasets during runtime in order to find simple behavior patterns and therefore predict an interval of values that represents an acceptable value. However, we de not want to keep previous data for each data point of the dataset we are monitoring, which incurs a $100 \%$ $400 \%$ memory overhead. Instead, we keep only minimal data for each dataset, in order to limit the memory footprint of our detector, while still providing a high SDC detection level. Therefore, we propose to compute the expected interval (i.e., range of values accepted as a correct data value) using statistics of the whole cluster of points (as opposed to per-point historical data). If the observed value is outside the expected interval, then it is considered an outlier. When an outlier is detected, an alarm is raised so that the application can mitigate the corruption. The mitigation strategy is given by the user. It could be to roll back to the last checkpoint, apply some application-specific data reconstruction technique, or simply log the SDC alert in a file for post-execution analysis.

The main idea is to gather statistical information for the whole cluster of points. In particular, we generate and monitor multiple distributions. The distributions are generated in several steps. First, we generate something that we call a field, which is extracted from the dataset (see Section 3.2). This field is never fully stored in memory. Instead, we start by extracting the minimum and maximum values of the field in the first iteration and we divide the interval [ $\min , \max ]$ into 256 segments of equal size. In the next iteration, we generate the new field and we count the number of values present in each of the 256 segments. We store this data in a small table (i.e., $256^{*} 4$ bytes $=1 \mathrm{~KB}$ ), which gives us the probability density function (PDF). We note that a field generated at time $t$ is expected to have a PDF similar to that generated at time $t-1$. However, a few values might be slightly outside the interval $[\min (t-1), \max (t-1)]$ (i.e., new minimum and/or new maximum). Thus, we allow the minimum and the maximum of the distribution to evolve with the execution. To this end, we keep the minimum and maximum of the two previous distributions, and we try to predict the new minimum and maximum using a linear interpolation. To allow for a certain margin of error in our prediction we add a small buffer $\varepsilon$ (e.g., 1\%). At time $t$, any point outside the distribution predicted at time $t-1$ is flagged as an outlier. If no outlier is found, the statistics gathered are used to predict the distribution at time $t+1$. We note that this procedure is done for each large dataset in each MPI rank. This monitoring-detection strategy is depicted in Figure 2(a).

\subsection{Four Types of SDC Detection}

Here we explain how we generate the fields from the dataset and what they represent. We use four levels of detection in the four different fields that exploit spatial and temporal smoothness properties. By exploiting different properties we guarantee a larger coverage. 


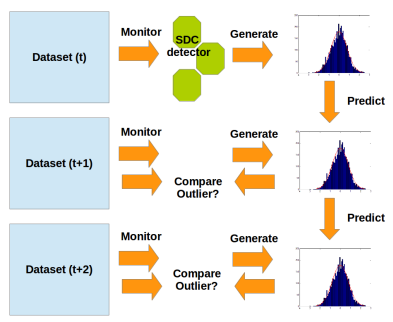

(a) Data distribution monitoring

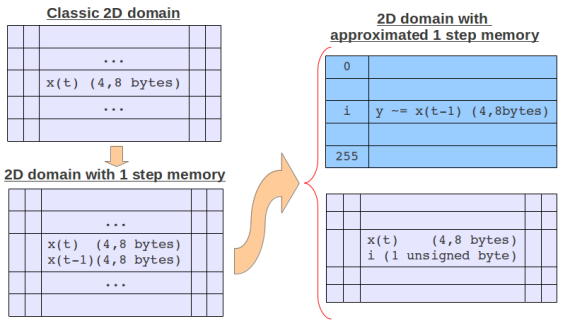

(b) Memory saving indexation strategy

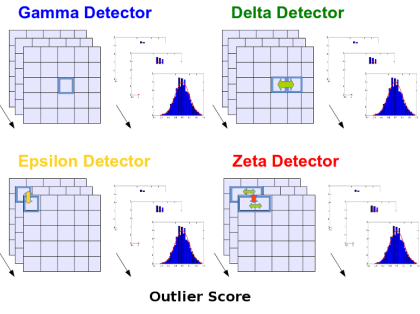

(c) Fuzzy logic module

Figure 2: Cluster-based online data monitoring multi-detector.

\section{Plain Detector.}

The first detector we propose takes the plain monitored dataset as the field and monitors its distribution to detect SDC. As an example, let us assume a dataset $\gamma$ is being monitored. Our detector will summarize the dataset in a small table representing the data distribution and will keep monitoring the evolution of that distribution. Any point outside that distribution is flagged as an outlier. We call this detector a $\gamma$-detector.

\section{Spatial Detector.}

The second type of detector that we propose analyzes the space variations of the dataset. This detector will compute at each time step $t$ a $\delta$ field as follows;

$$
\delta_{[i, j]}(t)=\gamma_{[i, j]}(t)-\gamma_{[g, h]}(t)
$$

where $\gamma_{[i, j]}$ and $\gamma_{[g, h]}$ are neighbor points following any of the axes of this 2D domain. This computation can take into account neighbors from multiple dimensions depending on the structure of the domain and the preferences of the user. This $\delta$ field represents the first-order spatial derivative of the monitored dataset. Then, the detector will produce and store the distribution of the $\delta$ field for the current time step $t$ and predict it for the time step $t+1$. Similar to the previous case, any point showing a $\delta_{[i, j]}(t+1)$ outside the expected distribution will be treated as an outlier. We call this detector a $\delta$-detector.

\section{Temporal Detector.}

The third type of detector is based on the temporal evolution of a dataset. More precisely, for each data point we compute the difference $\epsilon$ as follows.

$$
\epsilon_{[i, j]}(t)=\gamma_{[i, j]}(t)-\gamma_{[i, j]}(t-1)
$$

Then, similarly to the other detectors, we compute the distribution of $\epsilon$ at time step $t$ and predict the distribution at time $t+1$, so that any $\epsilon_{[i, j]}(t+1)$ outside the predicted distribution will be treated as an outlier. This $\epsilon$ field represents the first-order temporal derivative of the monitored dataset. However, computing the field $\epsilon(t)$ at instant $t$ requires saving $\gamma(t-1)$ in memory, which involves a large space overhead. To avoid such memory overhead, we use an indexing technique that stores only an index (one byte) pointing to the closest value in the $\mathrm{PDF}$, instead of storing the complete value (i.e., 4 or 8 bytes in single or double precision, respectively), as presented in Figure 2(b). We call this detector an $\epsilon$-detector.

\section{Spatiotemporal Detector.}

The fourth detector that we propose is a spatiotemporal detector that computes the time evolution $\zeta$ of the $\delta$ field computed by the $\delta$-detector, as follows.

$$
\zeta_{[i, j]}(t)=\delta_{[i, j]}(t)-\delta_{[i, j]}(t-1)
$$

Computing the time gradient of the space gradient gives us an idea of when a dataset increases or decreases its level of turbulence. In other words, the $\zeta$ field represents the timewise derivative of the spatial derivative computed by the $\delta$ field. Similarly to the temporal detector, one must keep previous $\delta$ values (i.e., at time $t-1$ ) in order to compute the time difference. Thus, we employ the same indexing technique to reduce the memory overhead of storing full floating-point values. We call this detector a $\zeta$-detector.

\subsection{Combining Detectors through Fuzzy Logic}

Here we present how we couple the four detectors to form a single entity that transmits clear messages to the application. The aim is to decrease the likelihood of false positives and increase the confidence in true. For the coupling we use a fuzzy logic module. In contrast with a binary detector that would return True or False to express whether an outlier was detected in the application datasets, fuzzy logic allows us to quantify how much a data point is outside the expected distribution. We then can normalize this deviation for all four detectors.

For instance, let us take the distance $D=$ maximum minimum of a given distribution as the normalizing distance. That is, if a point is at a distance $D$ from the distribution (e.g., $\gamma_{[i, j]}=$ maximum $+D$ ), then the detector reports an outlier score of $100 \%$. Note that each detector has a different distribution and hence a different distance $D$. Nonetheless, each detector will report an outlier score of $100 \%$ if the point is at $D$ distance from the distribution, regardless of the difference between distributions. We note that for the same point, different detectors are likely to give different outlier scores because corruptions have a different impact on temporal and spatial gradients. We note that fuzzy logic should not be mistaken with classic probabilities. A probability expresses (in this case) the uncertainty about a data point being an outlier or not, whereas the outlier score given by this fuzzy logic module expresses the degree to which a data point is not inside the expected distribution: there is no uncertainty about this degree.

Another feature that we propose together with this fuzzy detector is the capability to tune the fuzzy logic module, in order to give different weight to different detectors. By 


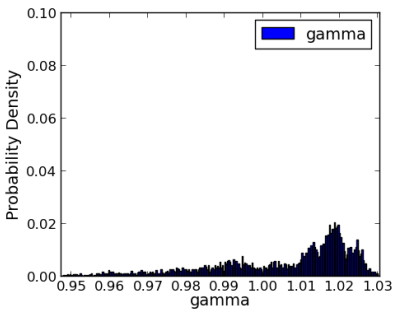

(a) $\gamma$-detector

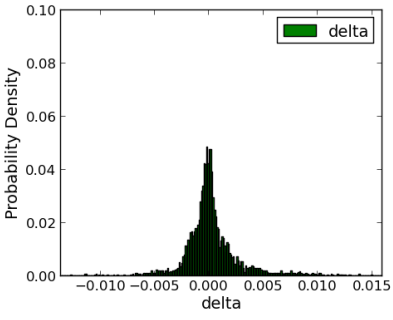

(b) $\delta$-detector

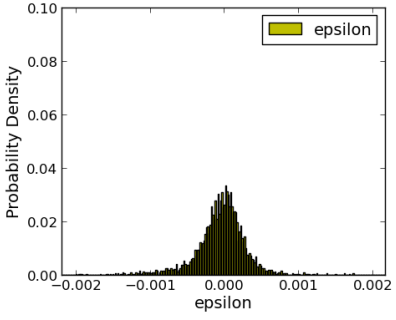

(c) $\epsilon$-detector

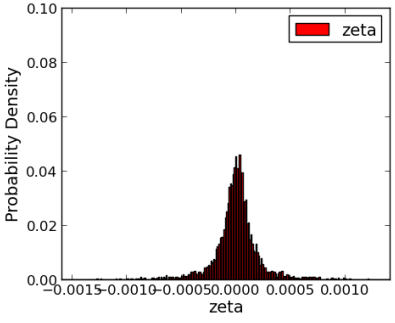

(d) $\zeta$-detector

Figure 3: Probability density function of gamma and delta detectors.

default, our detector will give same weight to all four detectors, but users may want to give more importance to a particular type of detector depending on the application or the input conditions. For instance, when the time-stepping resolution of a simulation is increased, one normally expects smaller changes from a time step to another (i.e., narrow distribution). Therefore it makes sense to give more weight to the $\epsilon$-detector in such a configuration. The same idea can applied to the $\delta$-detector when changing the scale and the space resolution of a simulation. By weighting the different detectors, users can completely remove a detector from the system. For instance, one could remove the $\zeta$-detector and the $\epsilon$-detector in order to minimize the memory overhead.

Giving an outlier score instead of a Boolean enables users to take different actions for different anomalies. For instance, a user could choose to ignore detections with less than $10 \%$ deviation, $\log$ into a file outliers with a score between $10 \%$ and $100 \%$, and trigger a roll back to the last checkpoint for any outlier with a deviation larger than $100 \%$.

\section{EVALUATION}

We implemented all the proposed detectors in the FTI library [2], so that applications need only to define the datasets to protect; all the detection work will be done automatically in a transparent fashion. Users can define the SDC checking frequency using a configuration file. Higher frequency decreases the detection latency, and lower frequency minimizes the computational overhead. For the evaluation we used the same CFD turbulent code presented in Section 2, and we monitor the velocity datasets of the application.

\subsection{Data Distribution of the Detectors}

First, we studied the distribution of the different detectors (see Section 3.2) at a given time step $t$ for a corruption-free execution. The first detector we examined was the $\gamma$-detector. Figure 3(a) shows the data distribution for the $u 1$ velocity field. As we can see, most values are between 1.00 and 1.03 . A smaller percentage of the dataset shows values under 1.0, which correspond to the areas close to the duct walls where the speed decreases because of friction. The distribution uses 256 bins in single precision, for a total size of $1 \mathrm{~KB}$ of data. From the plot we notice that the data is relatively well distributed across the spectrum of values and that none of the bins holds more than $2 \%$ of the data points. Given the statistical dispersion of the data and the wide interdecile range (IDR), the $\gamma$-detector is not expected to perform well for this dataset.

The second detector we analyzed was the $\delta$-detector. The distribution produced by the $\delta$ field is shown in Figure 3(b).
We can see a clear Gaussian distribution with 0 as the expectation and a low variance. We note that the dispersion of the $\delta$ distribution is low and its IDR is several times smaller than the IDR of the $\gamma$ distribution. Hence, it is more sensitive to data corruption. Thus, for this particular dataset, the $\delta$-detector is expected to perform better than the $\gamma$-detector.

The $\epsilon$-detector also shows a Gaussian distribution, although with slightly different varience from that of the $\delta$ distribution. Again, the expectation is 0.0 , and the variance is low. We note that the $\epsilon$ IDR is about one order of magnitude smaller than the IDR of the $\delta$ distribution. This is due to the high time resolution, low space resolution, and particular characteristics of the fluid (e.g., viscosity). Other executions of a similar CFD simulation with different input parameters could produce different distributions.

The $\zeta$-detector also produces a Gaussian distribution. The IDR is within the same order of magnitude but is narrower than those of the previous cases. We note that the large majority of values are within a small $\left(10^{-3}\right)$ segment. That is, any corruption producing a perturbation higher than $10^{-3}$ in this field will be detected by the $\zeta$-detector. Thus, for this dataset this detector is expected to show the highest recall among all four detectors.

\subsection{Performance Overhead}

Our goal is to propose an SDC detector with a low performance overhead, so that scientists can use these techniques in production runs. In order to achieve this goal, several implementation decisions were necessary. First, SDC detectors should monitor only local data, so no data transfers are involved. Second, grid borders and ghost regions should compare spatial differences only with internal cells; no comparison is done between cells located in different MPI processes. This step is, again, to avoid the need for extra communications. Also, the computation done for each grid point is minimal. Moreover, the frequency at which the SDC detector is triggered is a parameter set by the user.

We measured the time taken by our SDC detector and compared it with the time consumed by one iteration of the turbulence code presented previously. The results are plotted in Figure 4(a). As we can see, the time consumed by one application iteration is three orders of magnitude longer than the time consumed by our SDC detector to check the monitored datasets. This is not surprising because the application executes multiple kernels and performs several MPI communications at each iteration, which is a much heavier workload than what our SDC detector has. We note that in this experiment the SDC detector was executed at every iteration. Given this low verification time, it is not 


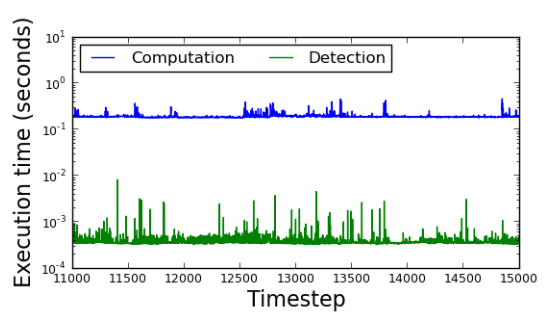

(a) Data monitoring performance

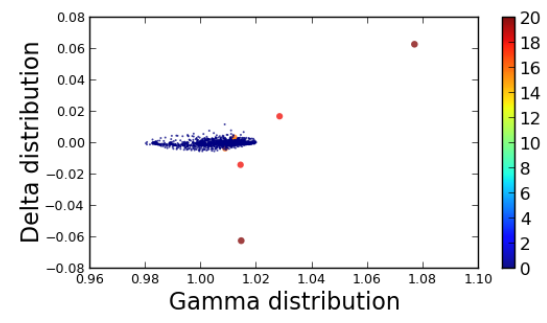

(b) $\gamma-\delta-$ cluster

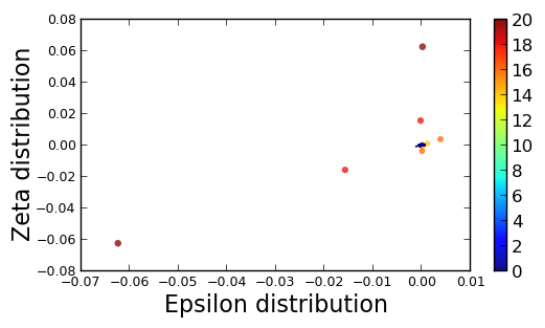

(c) $\epsilon-\zeta-$ cluster

Figure 4: Outlier detection with multiple detectors.

surprising that the overhead imposed by this approach is less than $1 \%$, hence, negligible.

\subsection{Outlier Detection}

Next, we want to verify that the detector can detect outliers according to the proposed scheme. The four distributions generated by the four detectors can be seen as a four-dimensional space where a dataset is represented by a cloud of points. Outliers would correspond to points far from that cloud and should be relatively easy to spot. To visualise how this detection mechanism works, we performed the following experiment. We ran the simulation together with the four detectors, which check for data corruption at every time step. Then, at time step $t$, we injected multiple bit-flips in different points of the domain, with different bit positions for each injection. Then, we ploted the detectors four-dimensional state just after the bit-flips were injected and the detectors had analyzed the datasets. We divided the four-dimensional space into a couple of two-dimensional hyperplanes with the clouds of points where it is easy to visualize the outliers. We colored each point depending on the level of corruption (i.e., the bit position corrupted) of each point (dark blue means no corruption).

As Figure 4(b) indicates, corruptions in the high bits of the mantissa (i.e., bits 18 and 20) generate clear outliers that are at a large distance from the $\gamma-\delta-$ cluster of noncorrupted points. We note that some corruptions are detected multiple times, once when analyzing the corrupted point and once more when analyzing the points around the corrupted one. For instance, in Figure 4(b) we see two corruptions in the upper-right part of the plot. These correspond to the actual corrupted data points because both $\delta$ and $\gamma$ are distant from the cluster. In the bottom part of the plot, we observe two corruptions complementing those two outliers. These are neighbors that have noncorrupted data (normal $\gamma$ ) but that do see an abnormal distance (abnormal $\delta$ ) from their corrupted neighbor. This phenomenona of the detection mechanism can help increase the confidence in a given outlier detection. However, we see that this does not help increase the detection of less critical corruptions (i.e., bits $16^{\text {th }}$ and $\left.14^{\text {th }}\right)$. Such corruptions are located in the limits of the $\gamma-\delta-$ cluster and are not detected as outliers.

Fortunately, the $\epsilon-\zeta-$ cluster does a much better job, positioning all those corruptions outside the cluster. In fact, the corruptions on the high bits of the mantissa generate such a large deviation that the entire cluster looks as a pale blue dot, as shown in Figure 4(c). We also observe the same multiple-detection phenomenon as in the previous case. The reason is that the $\zeta$-detector is also based on a spatial analysis, making neighbor points capable of detecting the corruption. This cluster visualization of the data distribution helps us understand the fuzzy logic module and the scoring system. The distance from the point to the cluster quantifies the level of deviation of the outlier.

\subsection{False Positives Study}

Detecting distant points as outliers is good only if the detector does not classify other noncorrupted points as outliers. That is, we need to quantify how many false positives our detectors observe during the execution. Since this approach classifies a point as outlier if is outside the expected distribution, the false positive rate will heavily depend on the capability of the detector to predict and adapt to the evolving data distribution. That is, the distribution elasticity allowed by our detector will play a critical role in minimizing the number of false positives. For our technique, this elasticity is represented by the parameter $\varepsilon$ (see Section 3.1 ).

To study the false positive rate of the SDC detector, we set up the following experiment: We set the allowed prediction error for all four distributions to $1 \%$ (i.e., $\varepsilon=1$ ), and we ran the turbulence code for a hundred iterations without injecting any corruption. Then, we counted the number of false positives and computed the false positive rate. We note that this rate can be higher than $100 \%$ if multiple false positives are detected within one iteration. Then, we repeated the experiment with an allowed prediction error of $2 \%$, and we kept increasing it up to $\varepsilon=10$. The results are plotted in Figure 5(a).

As we can see, the first three executions give a false positive rate of over a $100 \%$. That is, false outliers are found at every iteration. Then, as $\varepsilon$ increases up to $10 \%$, the false positive rate comes down to only $5 \%$. Although this is a better result than the results obtained with small $\varepsilon$, it is probably not sufficient to be used in production. For this miniapp that takes only a few seconds per iteration, having an outlier every twenty iterations is equivalent to seeing a false positive every minute. If the mitigation technique used for this run was rollback to the last checkpoint, the application could not make any real progress, since it would spend most of the time rolling back.

Thus, we explored a second approach in which the allowed prediction error is not given by the user but is learned during the first iterations of the execution. That is, we started with $\varepsilon=1$, and we allowed it to adapt dynamically during a certain learning period. After this time elapsed, $\varepsilon$ was blocked to its current value, which is the elasticity allowed for the rest of the execution. The learning period had to be long enough to get a good glimpse of the distribution 


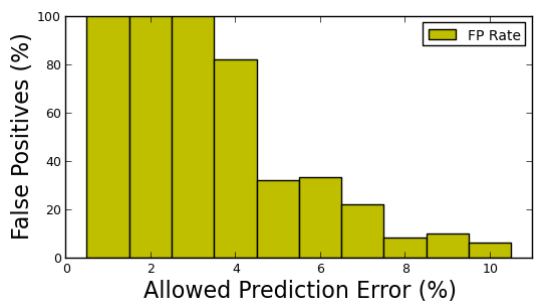

(a) False positives with static $\varepsilon$

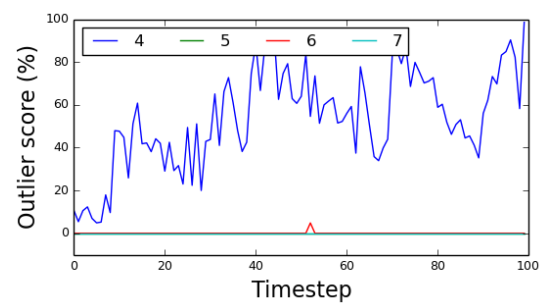

(b) False positives with dynamic $\varepsilon$

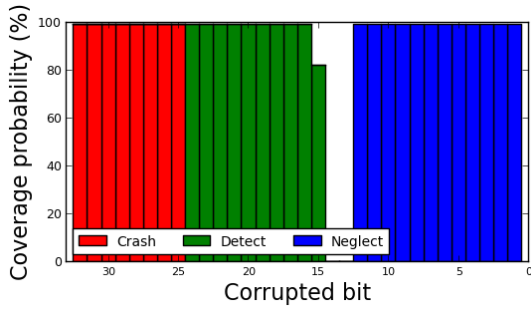

(c) SDC coverage

Figure 5: False Positive Rate and Detection Recall.

evolution, but short enough to minimize the risk of SDC during that time. We note that each of the four detectors has a different distribution and therefore the final $\varepsilon$ is likely to be different for each. To measure the false positive rate of this dynamic approach, we performed the following experiment: We set $\varepsilon=1$ for all detectors at the beginning of the execution, and we allowed it to change during the first four iterations of the execution. That is, any outlier observed during those iterations will be neglected and $\varepsilon$ readjusted to the outlier score of the presumed outlier. We chose four iterations because it is the minimum amount of time required to have all detectors working with a correct distribution (see Section 3.1). Then, we increased the learning period to 5,6 , and 7 iterations. Here, we want to show, not only the false positive rate but also the outlier score given by our detector. We recall that different mitigation measures can be taken depending on the outlier score. Thus, for each iteration, we plot the outlier score of the largest outlier detected in that iteration. We configured our application to ignore SDC alerts with an outlier score under $1 \%$.

The results are depicted in Figure 5(b). As we can see, a learning period of four iterations is not enough, since false positives are raised at every iteration, sometimes with outlier scores of over $80 \%$. Fortunately, increasing the learning period dramatically decreases the false positive rate. Indeed, only one false positive is detected with learning periods of 5 and 6 iterations, and the outlier score is less than $5 \%$. With a learning period of 7 iterations, we do not see any false positive for this turbulence code. The reason for such a dramatic reduction of false positives is that all four detectors understand quickly at which speed (i.e., distribution elasticity) the distributions evolve, and they incorporate this knowledge in their parameters. The final values of $\varepsilon$ were $1.0,3.6,6.3$, and 13.3 for the $\gamma, \delta, \epsilon$, and $\zeta$ detectors, respectively. Not surprisingly, the $\zeta$-detector requires more elasticity than the others, since it is the most accurate for this application. We note that the fuzzy logic module reduces the number of false positives by weighting the score with those of the other detectors. In particular a few times one detector noticed a false outlier with an outlier score between $0.5 \%$ and $3.5 \%$, but the other detectors did not consider it as an outlier (i.e., correct classification). The final score, being the weighted sum of all the scores, was under $1 \%$ and was neglected. This proves that our detector can achieve over $99 \%$ of detection precision with the right configuration. We use this configuration for the rest of the evaluation.

\subsection{Detection Recall}

After obtaining a false positive rate lower than $1 \%$ we want to study how much SDC detection can achieve this technique on this configuration. Clearly, allowing for some elasticity to decrease false positives might have a bad impact on the detection recall. Thus, we need to know whether our SDC detector can still guarantee a good recall. Hence, we ran an experiment in which corruptions were injected at random points in time, at random positions in the domain and at random bit positions. All injections were logged in order to compare the injections and the detections with a postmortem script. For the first experiments we used the familiar turbulent flow code. We set the allowed error to $10^{-4}$ so corruptions in the last 12 bits of the mantissa could be neglected (see Section 2.2). Corruptions on the 8 most significant bits make the application unstable, and it crashes. As shown in Figure 5(c), our detectors can detect almost all corruptions occurring in bits $16^{\text {th }}$ and above and about $82 \%$ of corruptions in bit $15^{\text {th }}$. Below that point, the detector cannot see any other perturbation as abnormal.

For more detailed analysis, we recorded the outlier score given by the detector to each one of the corruptions injected. Then, we computed the average outlier score for each bit position. In particular, we focus on the transition between detected bit-flips to nondetected bit-flips. Our study shows that the average outlier scores are $0 \%, 14 \%, 72 \%$, and $217 \%$ for bit positions $14^{t h}, 15^{t h}, 16^{t h}$, and $17^{t h}$, respectively. These results confirm that corruption affecting bits $16^{\text {th }}$ and above are clearly seen as outliers. For bit $15^{\text {th }}$ the outlier score will depend on the location in the domain. Corruption in slightly more turbulent areas could pass undetected for bit $15^{t h}$. In this work, we try to protect the vulnerable bit positions, that is the bit positions that do not produce a crash when corrupted and that produce a non-negligible error for the application. For this application, that corresponds to bit positions $13^{\text {th }}$ to $25^{\text {th }}$. From this range of vulnerable bits, our detector can detect most corruptions, at the exception of corruptions stinking in bits $13^{\text {th }}, 14^{\text {th }}$ and partially $15^{\text {th }}$. We call those corruptions Catastrophic Corruptions because they produce a significant error in the application and they cannot be detected by this detector. Given the vulnerable bit positions presented above, we can estimate the probability of a corruption to be catastrophic $P_{C C}$ at about $20 \%$. Overall, this technique detects about $80 \%$ of SDC occurring in the vulnerable bits.

\subsection{Multi-bit Corruptions}

Up to this point, we have assumed that SDC corruptions always corrupt one single bit, which might not be always the case. In fact, ECC memories can detect all single bit and detect double bit corruptions, hence if SDC happens in ECC 


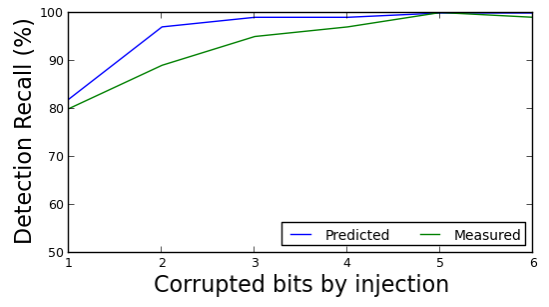

(a) Detection recall for multi-bit errors

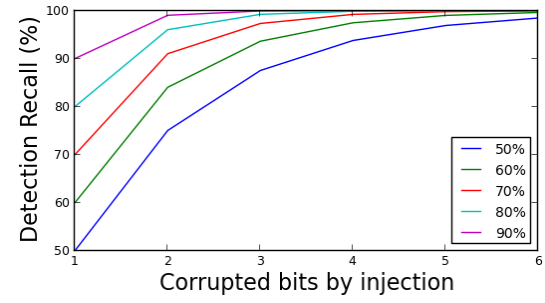

(b) Recall for different single-bit recalls

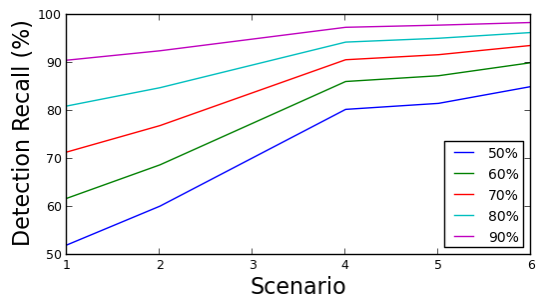

(c) Recall for mixed types of corruption

Figure 6: SDC detection recall for multi-bit corruptions.

protected memories, it is sure that those errors corrupt more than two bits. In addition, corruptions occurring inside the processing units might propagate as multi-bit corruptions. Therefore, in this section we explore how our detector behaves in the presence of multi-bit corruptions.

Using a probabilistic approach, we can predict the recall for multi-bit errors based on the recall of single-bit corruptions. Instead of computing the probability of detection, we compute the probability of non detection, in other words, the probability $P_{C C}$ for an SDC to be a catastrophic corruption. Now, if we consider multi-bit errors, the probability of such corruption to be catastrophic is equivalent to the probability of all of those bit corruptions to be catastrophic. In a formal way, if we consider a multi-bit corruption affecting $b$ bits, the probability for that corruption to be catastrophic is $P_{C C}^{b}$.

Based on this formal prediction of SDC detection for multibit errors, we perform an experiment trying to verify it with experimentation. Thus, we modify our SDC injector to corrupt an arbitrary number of bits $b$ in each corruption, where $b$ is passed as parameter to the injector. Then, we run our CFD turbulent code and inject single-bit errors in the vulnerable bits during runtime and we measure the detection recall. We perform the same run eight times to get a good estimate and we report the average detection recall. We repeat the experiment for double-bit errors, triplebit corruptions and so on up to 6 -bit errors. The results are depicted in Figure 6(a). The first thing we observe, is that, as expected, the detection recall increases as the number of corrupted bits increases. Then, we notice that the predicted recall is slightly better than the measured recall for low number of corrupted bits, but it quickly approaches the predicted recall for higher number of bits corrupted.

Given the accuracy of the predicted recall, we can explore what would be the multi-bit detection recall for applications that expose different single-bit detection recalls. In Figure 6(b) we can see that the detection recall increases fast as the number of the corrupted bits increases, and this for all initial single-bit detection recalls. For instance, from 4-bit corruptions, all cases show a multi-bit detection recall over $90 \%$. This results demonstrate that even applications with very chaotic data that expose a relatively low single-bit detection recall, could significantly profit from this technique and get high detection recall in systems where the multi-bit errors are more prone than single-bit corruptions.

Knowing the detection recall for multi-bit corruption is interesting but we cannot assume that all corruptions always corrupt the same number of bits. It is more realistic to assume that single-bit and multi-bit errors can happen with different levels of probability. Therefore, we study six different scenarios with different corruption distributions, as shown in Table 1 . Then, we study the overall SDC detection recall given the different probabilities of multi-bit data corruption of the given scenarios. The results are depicted in Figure 6(c). Again, we plot the results based on the initial detection recall for single-bit corruptions. As we can see, as the probability of multi-bit corruption increases with the different scenarios, the detection recall increases, to the point that for scenario 5 and 6 , the overall detection recall is over $80 \%$, even for applications with a single-bit recall of $50 \%$.

Table 1: Multi-bit Corruption Scenarios

\begin{tabular}{c|c|c|c|c|c|c} 
scenario & 1-bit & 2-bit & 3-bit & 4-bit & 5-bit & 6-bit \\
\hline 1 & 0.95 & 0.01 & 0.01 & 0.01 & 0.01 & 0.01 \\
2 & 0.75 & 0.05 & 0.05 & 0.05 & 0.05 & 0.05 \\
3 & 0.50 & 0.10 & 0.10 & 0.10 & 0.10 & 0.10 \\
4 & 0.25 & 0.15 & 0.15 & 0.15 & 0.15 & 0.15 \\
5 & 0.20 & 0.20 & 0.15 & 0.15 & 0.15 & 0.15 \\
6 & 0.15 & 0.15 & 0.15 & 0.15 & 0.20 & 0.20
\end{tabular}

In summary, our evaluation showed that the overhead over the application is negligible (i.e., less that 1\%) and that the false positive rate can be reduced to less than $1 \%$ too. In addition, we demonstrated that our SDC detector reaches over $80 \%$ of detection recall on the turbulent CFD code studied. Moreover, the detection recall increases to over $95 \%$ when we take into account multi-bit corruptions.

\section{RELATED WORK}

Soft errors and data corruption for extreme-scale computers have been the target of numerous studies. Mitigation techniques have been proposed at multiple layers of the architecture offering different levels of coverage and overhead trade-off for each of them. An ideal SDC detection technique should be easily applicable to a wide spectrum of HPC applications and should have a low false positive rate, while incurring a minimum performance cost over the application.

\subsection{Hardware-Level Detection}

Some scientists are working on solving the problem of data corruption at the hardware level. This method is appealing in that applications do not require any adaptation to benefit (i.e., it is transparent to the application). Considerable literature exists on soft errors rates $[19,20,5,9]$ and detection techniques at the hardware level [18, 11]. Implementing these techniques efficiently is difficult, however, under the strict constraints of extreme-scale computing (e.g., low power consumption). Designing robust systems to guarantee low 
error rate requires extra power and extra chip area. Some studies have shown that the SER can be substantially reduced without too much overhead $[17,16]$. Even if such requirements could be met, however, it is unclear whether the market will drive the technologies in this direction.

\subsection{Process Replication}

Process replication has been used for many years to guarantee correctness in critical systems, and its application to HPC systems has been studied. For example, doubleredundant computation can detect SDC by comparing the messages transmitted between the replicated processes [12] Also, triple redundancy can be used to enable data correction using a voting scheme. This approach is general in that applications need relatively little adaptation to benefit from double or triple redundancy. Unfortunately, doubleand triple-redundant computation always imposes large overheads, since the number of hardware resources required doubles or triples. In addition, the cost of energy consumption is heavily increased when sending every message two or three times. In order to avoid such overhead, partial replication [21] has been proposed and evaluated on task-based models, showing that it offers a large coverage for a low replication rate.

\subsection{Algorithm-Based Fault Tolerance}

A promising technique against data corruption is algorithmbased fault tolerance (ABFT) [13]. This technique uses extra checksums in linear algebra kernels in order to detect and correct corruptions $[13,10,7,6]$. It imposes a low overhead on the application and guarantees a good SDC detection recall in general. Some recent studies [8] have shown that ABFT can be implemented for matrix multiplications on hardware accelerators. In addition to its detection capability, ABFT offers correction features. However, ABFT has been implemented only on some linear algebra kernels, a small subset of the vast spectrum of computational kernels. Moreover, this technique is not general: each algorithm needs to be adapted for ABFT. Furthermore, even applications that employ only ABFT-protected kernels can fail to detect SDCs if the corrupted data lies outside the protected regions.

\subsection{Approximate Computing}

Another type of SDC detection is based on the idea of approximate computing, in which a computing kernel is paired with a cheaper and less accurate kernel that will produce close enough results that can be compared with those generated by the main computational kernel [3]. This detection mechanism has shown promising results but is still not general enough, since each application will need to manually be complemented with the required approximate computing kernels. Furthermore, complex applications will need to adapt multiple different kernels with a second-order verification kernel in order to offer good coverage.

\subsection{Our Previous Data Monitoring Detectors}

Our previous work, studied spatial smoothness [1] and time series [4]. Spatial smoothness guaranteed a low overhead but showed partial results. Time series showed better detection recall but imposed between $200 \%$ to $400 \%$ of memory overhead per data point. In this work, we mix spatial and temporal analysis and we complement them using a fuzzy logic module. This is our first work that combines multiple types of detectors (i.e., spatial and temporal) to improve detection recall and decrease false positive rate.

\section{CONCLUSION}

In this work we have studied some of the properties of HPC applications and their datasets. Based on these properties, we proposed several SDC detectors that analyze the datasets of an application and generate the next expected distribution in order to detect anomalies. To integrate all the detectors together, we proposed a fuzzy logic module that gives detailed information to the user about the detected anomalies. The fuzzy logic module, not only fuses all the detectors but also provides an outlier score so that the application can take different mitigation strategies depending on the type of error. We reduce the false positive rate dramatically by using an elasticity parameter that learns during the first iterations of the execution and adapts dynamically. In addition, several optimizations were introduced (e.g. an indexing strategy) to reduce overhead to a negligible level.

We evaluated our proposed scheme with a turbulent flow simulation based on Navier-Stokes equations. We studied the propagation of corruption across the entire domain and for different levels of corruption. We analyzed the distributions generated by our four detectors and demonstrated their detection accuracy using a cluster visualization technique. We performed a false positive study and proposed a dynamic elastic approach to dramatically reduce the false positive rate to under $1 \%$. The results show that our technique can detect over $85 \%$ of corruptions while incurring only negligible overhead (less than 1\%) on the scientific application. We demonstrated that with the help of these detectors, applications can have up to $90 \%$ of coverage against SDC.

As future work, we plan to implement subregion decomposition inside each MPI rank, in order to increase further the detection recall of our SDC detector.

\section{Acknowledgments}

This work was supported by the U.S. Department of Energy, Office of Science, Advanced Scientific Computing Research Program, under Contract DE-AC02-06CH11357; and by the ANR RESCUE, the INRIA-Illinois-ANL-BSC Joint Laboratory on Extreme Scale Computing, and Center for Exascale Simulation of Advanced Reactors (CESAR) at Argonne.

\section{REFERENCES}

[1] L. Bautista Gomez and F. Cappello. Detecting silent data corruption through data dynamic monitoring for scientific applications. In Proceedings of the 19th ACM SIGPLAN Symposium on Principles and Practice of Parallel Programming, PPoPP '14, pages 381-382, New York, NY, USA, 2014.

[2] L. A. Bautista-Gomez, S. Tsuboi, D. Komatitsch, F. Cappello, N. Maruyama, and S. Matsuoka. FTI: High performance fault tolerance interface for hybrid systems. In $S C$, page 32 . ACM, 2011.

[3] A. R. Benson, S. Schmit, and R. Schreiber. Silent error detection in numerical time-stepping schemes. International Journal of High Performance Computing Applications, page 1094342014532297, 2014.

[4] E. Berrocal, L. Bautista-Gomez, S. Di, Z. Lan, and F. Cappello. Lightweight silent data corruption 
detection based on runtime data analysis for hpc applications (to appear). In The ACM International Symposium on High-Performance Parallel and Distributed Computing (HPDC), HPDC '15. ACM, 2015.

[5] S. Borkar. Designing reliable systems from unreliable components: The challenges of transistor variability and degradation. IEEE Micro, 25:10-16, November 2005.

[6] G. Bosilca, A. Bouteiller, T. Herault, Y. Robert, and J. Dongarra. Assessing the impact of abft and checkpoint composite strategies. In 2014 IEEE International Parallel \& Distributed Processing Symposium Workshops (IPDPSW), pages 679-688. IEEE, 2014.

[7] G. Bosilca, A. Bouteiller, T. Herault, Y. Robert, and J. Dongarra. Composing resilience techniques: Abft, periodic and incremental checkpointing. International Journal of Networking and Computing, 5(1):2-25, 2015.

[8] C. Braun, S. Halder, and H. J. Wunderlich. A-abft: Autonomous algorithm-based fault tolerance for matrix multiplications on graphics processing units. In 2014 44th Annual IEEE/IFIP International Conference on Dependable Systems and Networks (DSN), pages 443-454. IEEE, 2014.

[9] A. Cataldo. Mosys, iroc target ic error protection, 2002.

[10] Z. Chen. Online-ABFT: an online algorithm based fault tolerance scheme for soft error detection in iterative methods. In Proceedings of the 18th ACM SIGPLAN symposium on Principles and Practice of Parallel Programming, pages 167-176. ACM, 2013.

[11] T. J. Dell. A white paper on the benefits of Chipkill-correct ECC for PC server main memory. IBM Microelectronics Division, pages 1-23, 1997.

[12] D. Fiala, F. Mueller, C. Engelmann, R. Riesen, K. Ferreira, and R. Brightwell. Detection and correction of silent data corruption for large-scale high-performance computing. In Proceedings of the International Conference on High Performance Computing, Networking, Storage and Analysis, page 78. IEEE Computer Society Press, 2012.

[13] K.-H. Huang and J. A. Abraham. Algorithm-based fault tolerance for matrix operations. IEEE Transactions on Computers, 100(6):518-528, 1984.

[14] IEEE. IEEE Standard for Floating-Point Arithmetic. http://standards.ieee.org/findstds/standard/7542008.html.

[15] D. Li, J. S. Vetter, and W. Yu. Classifying soft error vulnerabilities in extreme-scale scientific applications using a binary instrumentation tool. In Proceedings of the International Conference on High Performance Computing, Networking, Storage and Analysis, page 57. IEEE Computer Society Press, 2012.

[16] Y. Li, S. Makar, and S. Mitra. Casp: concurrent autonomous chip self-test using stored test patterns. In Proceedings of the Conference on Design, Automation and Test in Europe, pages 885-890. ACM, 2008.

[17] S. Mitra, K. Brelsford, Y. M. Kim, H.-H. Lee, and Y. Li. Robust system design to overcome cmos reliability challenges. IEEE Journal on Emerging and Selected Topics in Circuits and Systems, 1(1):30-41, 2011.
[18] S. S. Mukherjee, J. Emer, and S. K. Reinhardt. The soft error problem: An architectural perspective. In 11th International Symposium on High-Performance Computer Architecture., pages 243-247. IEEE, 2005.

[19] E. Normand. Single event upset at ground level. IEEE Transactions on Nuclear Science, 43(6):2742-2750, 1996.

[20] T. Semiconductor. Soft errors in electronic memory - a white paper, 2004.

[21] O. Subasi, J. Arias, J. Labarta, O. Unsal, and A. Cristal. Leveraging a task-based asynchronous dataflow substrate for efficient and scalable resiliency. In Workshop on Dependable Multicore and Transactional Memory Systems. DMTM, 2014.

The submitted manuscript has been created by UChicago Argonne, LLC, Operator of Argonne National Laboratory ("Argonne"). Argonne, a U.S. Department of Energy Office of Science laboratory, is operated under Contract No. DEAC02-06CH11357. The U.S. Government retains for itself, and others acting on its behalf, a paid-up, nonexclusive, irrevocable worldwide license in said article to reproduce, prepare derivative works, distribute copies to the public, and perform publicly and display publicly, by or on behalf of the Government. 